\title{
ON THE TOEPLITZ ALGEBRAS OF RIGHT-ANGLED AND FINITE-TYPE ARTIN GROUPS
}

\author{
JOHN CRISP and MARCELO LACA
}

(Received 19 May 2000; revised 21 March 2001)

Communicated by G. Willis

\begin{abstract}
The graph product of a family of groups lies somewhere between their direct and free products, with the graph determining which pairs of groups commute. We show that the graph product of quasi-lattice ordered groups is quasi-lattice ordered, and, when the underlying groups are amenable, that it satisfies Nica's amenability condition for quasi-lattice orders. The associated Toeplitz algebras have a universal property, and their representations are faithful if the generating isometries satisfy a joint properness condition. When applied to right-angled Artin groups this yields a uniqueness theorem for the $C^{*}$-algebra generated by a collection of isometries such that any two of them either *-commute or else have orthogonal ranges. The analogous result fails to hold for the nonabelian Artin groups of finite type considered by Brieskorn and Saito, and Deligne.
\end{abstract}

2000 Mathematics subject classification: primary 20F36, 22D25, 46L05, 46L55, 47B35.

Keywords and phrases: graph product, quasi-lattice order, covariant isometric representation, Toeplitz algebra, Artin group.

\section{Introduction}

Several celebrated results in $C^{*}$-algebra theory assert that the $C^{*}$-algebra generated by a semigroup of isometries does not depend on the specific isometries, provided they satisfy a properness condition. The situations described by these results are of considerable interest, stemming from the fact that the algebraic structure given by the semigroup operation determines a unique $C^{*}$-norm on the $*$-algebra generated by the isometries. As examples we have Coburn's theorem on the $C^{*}$-algebra generated by a

\footnotetext{
The first author was supported by an EPSRC Research Assistantship at the University of Southampton, United Kingdom, and a Postdoctoral Grant from the Conseil Regional de Bourgogne, France; he also wishes to thank the Department of Mathematics at the University of Newcastle, NSW, for their hospitality on numerous occasions. The second author was supported by the Australian Research Council.

(C) 2002 Australian Mathematical Society $1446-7887 / 2002 \$ A 2.00+0.00$
} 
single isometry [4], Douglas' theorem on the $C^{*}$-algebra of a one parameter semigroup of isometries [11], and the generalization by Murphy to the Toeplitz $C^{*}$-algebra of a totally ordered group [17]; in all these cases the properness condition simply says that the isometries are not unitary.

Moving away from total orders on abelian groups, Nica [18] considered a class of partially ordered groups $(G, P)$ he called quasi-lattice ordered. Inspired by what happens with the left regular (Toeplitz) representation of the positive cone $P$, he isolated a key covariance condition, which is automatic for total orders, and defined a universal $C^{*}$-algebra $C^{*}(G, P)$ whose representations are given by the covariant isometric representations of $P$. He proved that the uniqueness of the $C^{*}$-algebra generated by a covariant isometric representation depends on an amenability property of the quasi-lattice order that is strictly weaker than amenability of the underlying group. Indeed, he showed that Cuntz's result [7] on the uniqueness of the $C^{*}$-algebra $\mathscr{T} \mathscr{O}_{n}$ generated by $n$ isometries with orthogonal ranges can be seen as an amenability result for the canonical quasi-lattice order on the free group on $n$ generators. In this case the covariance condition requires that the generating isometries have orthogonal ranges, and the properness condition says that the sum of these ranges is not the whole Hilbert space.

In [16] Laca and Raeburn associated a semigroup dynamical system to each quasilattice order and showed that the corresponding crossed product is canonically isomorphic to the universal $C^{*}$-algebra $C^{*}(G, P)$. This approach led to two main advances. The first one was the generalisation to all quasi-lattice orders of some key estimates of Cuntz [6], which provides a convenient framework in which to study faithfulness of representations and uniqueness properties. The second one was a direct proof of the amenability of the quasi-lattice orders on a large class of (nonamenable) free product groups, which widened the range of application of the uniqueness results.

Direct products and free products of groups are both special cases of the more general construction of a graph product of groups (see Section 2 below), and in this paper we address the natural questions of whether graph products support quasilattice orders, and under which conditions these quasi-lattice orders are amenable in the sense of Nica [18]. Our main technical results are Theorem 10, which shows that graph products of quasi-lattice ordered groups are indeed quasi-lattice ordered, and Theorem 20, which gives a sufficient condition for their amenability. Combining this with the results of [16] in Theorem 21, we characterize faithful representations and give a uniqueness result for the Toeplitz algebras of graph products.

An interesting class of examples is that of graph products of copies of $(\mathbb{Z}, \mathbb{N})$, otherwise known as graph groups or right-angled Artin groups. It follows from our main results that, in the language of Nica [18], they are all amenable quasi-lattice orders, giving a unified statement of the amenability of the canonical quasi-lattice orders on all free groups and all free abelian groups, as well as providing many new 
examples of amenable quasi-lattice orders. The corresponding Toeplitz $C^{*}$-algebras are thus universal and unique. We state this main result in terms of generators and relations in Theorem 24, which contains, as extreme cases, Cuntz's theorem (in which the generating isometries have mutually orthogonal ranges) and a multivariable version of Coburn's theorem, (in which the generating isometries $*$-commute, that is, they commute with each other and with each other's adjoints). See [20] for results related to this last situation.

Other interesting quasi-lattice orders are provided by the family of Artin groups of finite type, with the embedded Artin monoid as positive cone $[1,10]$. These examples, which include the braid groups, are lattice groups, because every pair of elements has a least common upper bound. In Section 6 we prove, using an argument essentially due to Nica, that if a group is lattice ordered and amenable as a quasi-lattice order, then the group itself has to be amenable. Thus, in contrast to what happens with graph products, only the Artin groups of finite type that are amenable (and hence abelian) give rise to amenable quasi-lattice orders. The nonabelian Artin groups of finite type appear then as an important class of groups having canonical non-amenable quasilattice orders. As a consequence, the $C^{*}$-algebra generated by a covariant isometric representation depends, in general, on the specific representation, Theorem 30.

\section{Graph products of groups}

Graph products were defined in the thesis of Green [13], and have been subsequently studied by various other authors. We refer the reader to [14] and the references therein for further background.

Let $\Gamma$ denote a graph with vertex set $\Lambda$, and edge set $E(\Gamma) \subseteq\{\{I, J\}: I, J \in$ $\Lambda$ and $I \neq J\}$; the edges of $\Gamma$ have no orientation and there is no edge joining a point to itself. We say that vertices $I$ and $J$ are adjacent in $\Gamma$ if there is an edge $\{I, J\} \in E(\Gamma)$. Note that a vertex is never adjacent to itself. Given a family $\left\{G_{I}\right\}_{I \in \Lambda}$ of groups, we define the graph product $\Gamma_{I \in \Lambda} G_{I}$ to be the quotient of the free product $*_{\Lambda} G_{l}$ by the smallest normal subgroup containing the elements $x_{1} x_{2} x_{1}^{-1} x_{2}^{-1}$ for all pairs $x_{1} \in G_{I}, x_{2} \in G_{J}$ where $I$ and $J$ are adjacent in $\Gamma$. When the $G_{I}$ are all copies of $\mathbb{Z}$, the graph product is called a graph group, or right-angled Artin group. We shall not need to assume that $\Gamma$ is finite.

Suppose, for the rest of this section, that we are given a graph $\Gamma$, as above, and groups $\left\{G_{I}\right\}_{I \in \Lambda}$, and let $G=\Gamma_{I \in \Lambda} G_{I}$ denote the graph product. We may take as a generating set for $G$ the set

$$
\mathbb{G}=\coprod_{I \in \Lambda} G_{I} \backslash\{1\}
$$

Given $x \in \mathbb{G}$ we write $I(x)$ for the unique vertex $I$ such that $x \in G_{l}$. We say that $x$ 
belongs to $I(x)$.

By an expression $X$ for an element $x \in G$ we mean a word in the generators $\mathbb{G}$ representing $x$. Given an expression $X=x_{1} x_{2} \cdots x_{l}$, the elements $x_{i}$ are called syllables of $X$ and the number $l$ is called the length of $X$, written $l=\ell(X)$. We say that $l \in \Lambda$ is a vertex of $X$ if $I=I\left(x_{i}\right)$ for $x_{i}$ a syllable of $X$.

Given an expression $X=x_{1} x_{2} \cdots x_{l}$ for $x \in G$, the graph product relations allow one to modify $X$ to obtain a different expression for $x$ by replacing a subexpression $x_{i} x_{i+1}$ with $x_{i+1} x_{i}$ if $I\left(x_{i}\right)$ is adjacent to $I\left(x_{i+1}\right)$. In the terminology of [14], such a substitution is called a shuffle, and we shall say that two expressions are shuffle equivalent if one may be obtained from the other via a finite sequence of shuffles. If the expression $X$ contains a subexpression of the form $x_{i} x_{i+1}$, with $I\left(x_{i}\right)=I\left(x_{i+1}\right)$, then we may give a shorter expression for $x$ by an amalgamation, that is by deleting $x_{i} x_{i+1}$ in the case that $x_{i+1}=x_{i}^{-1}$ or otherwise by replacing the two syllables $x_{i} x_{i+1}$ with the single syllable $\hat{x}_{i} \in \mathbb{G}$ such that $\hat{x}_{i}=x_{i} x_{i+1}$.

We say that an expression is reduced if it is not shuffle equivalent to any expression which admits an amalgamation.

LEMMA 1. Given an expression $X=x_{1} x_{2} \cdots x_{l}$, the following are equivalent:

(i) $X$ is reduced;

(ii) for all $i<j$ such that $I\left(x_{i}\right)=I\left(x_{j}\right)$ there exists $k$ such that $i<k<j$ and $I\left(x_{k}\right)$ is not adjacent to $I\left(x_{i}\right)$.

Proof. That (ii) is a consequence of (i) is obvious. We note that the truth of condition (ii) is invariant under shuffles, while an amalgamation can never be performed on an expression satisfying (ii). Thus (ii) implies (i).

Given an expression $X=x_{1} x_{2} \cdots x_{1}$ for $x \in G$, one may easily produce a reduced expression for $x$ via a finite process of shuffling and amalgamating. If $X$ fails condition (ii) of Lemma 1 , that is, there exists $i<j$ such that $I\left(x_{i}\right)=I\left(x_{j}\right)$ and $I\left(x_{i}\right)$ commutes with $I\left(x_{k}\right)$ for all $i<k<j$, then an obvious sequence of shuffles will allow the syllables $x_{i}$ and $x_{j}$ to be amalgamated, reducing the length of $X$. One may continue reducing the length in this manner until condition (ii) is satisfied. The following theorem, due to Green [13], reduces the solution of the word problem in a graph product of groups to the solution of the word problem in each of the component groups (see [14] for an equivalent formulation).

THEOREM 2 (Green [13]). Any two reduced expressions for the same element of $G$ are shuffle equivalent.

As a simple consequence of Theorem 2 we may define the length $\ell(x)$ of an element $x \in G$ to be the length of any reduced expression $X$ for $x$, note that $\ell(x)$ is also the minimal length of any expression for $x$. If $X$ is a reduced expression for $x$, we are 
also justified in referring to a syllable or a vertex of $X$ as a syllable of $x$ or a vertex of $x$, respectively.

Let $X=x_{1} x_{2} \cdots x_{l}$ be a reduced expression, and $x_{i}$ any syllable of $X$. If $I\left(x_{i}\right)$ is adjacent to $I\left(x_{j}\right)$ for all $j<i$ then we say that $x_{i}$ is an initial syllable of $X$, and that $I\left(x_{i}\right)$ is an initial vertex of $X$. We write $\Delta(X)$ for the set of all initial vertices of $X$. The following facts are easily checked.

LEMMA 3. Let $X=x_{1} x_{2} \cdots x_{l}$ be a reduced expression.

(i) If $x_{i}$ is an initial syllable of $X$, then $X$ is shuffle equivalent to the expression

$$
x_{i} x_{1} \cdots x_{i-1} x_{i+1} \cdots x_{l}
$$

(ii) The initial vertices of $X$ are pairwise adjacent.

(iii) For each $I \in \Delta(X)$ there exists a unique initial syllable of $X$ belonging to $I$. We define the function $\delta_{X}: \Delta(X) \rightarrow \mathbb{G}$ such that $\delta_{X}(I)$ is equal to the initial syllable of $X$ belonging to $I$.

(iv) If $X^{\prime}$ is another expression which is shuffle equivalent to $X$, then $\Delta\left(X^{\prime}\right)=$ $\Delta(X)$ and $\delta_{X^{\prime}}=\delta_{X}$.

By virtue of Lemma 3 (iv) and Theorem 2 we may make the following definitions.

DEFINITION 4. Let $x \in G$. We define the set of initial vertices of $x$ to be the set $\Delta(x)=\Delta(X)$ where $X$ is any reduced expression for $x$. We also define, for each $I \in \Lambda$, the element $x_{I}$ to be the initial syllable of $x$ that belongs to $I$ if $I$ is an initial vertex of $x$, and 1 if not; that is,

$$
x_{I}= \begin{cases}\delta_{X}(I) & \text { if } I \in \Delta(x) ; \\ 1 & \text { if } I \notin \Delta(x) .\end{cases}
$$

Given an expression $X=x_{1} x_{2} \cdots x_{l}$ let $\operatorname{rev}(X)$ denote the expression $x_{l} x_{l-1} \cdots x_{1}$. This allows us to define the set of final vertices of $x \in G$ to be the set $\Delta^{r}(x)=$ $\Delta(\operatorname{rev}(X))$, where $X$ is any reduced expression for $x$. Similarly also, for each $I \in \Lambda$, we define the element

$$
x_{I}^{r}= \begin{cases}\delta_{\operatorname{rev}(X)}(I) & \text { if } I \in \Delta^{r}(x) ; \\ 1 & \text { if } I \notin \Delta^{r}(x),\end{cases}
$$

where $X$ is any reduced expression for $x$.

Observe that, by using Lemma 3 (i) and (ii), one may always find, for any given element $x \in G$, a reduced expression which begins with the product, in any order, of the $x_{I}$ for $I \in \Delta(x)$. Equally, one may always find a reduced expression for $x$ which ends with the product, in any order, of the $x_{I}^{r}$ for $I \in \Delta^{r}(x)$. 
LemMA 5. Given $x, y \in G$, let $D=\Delta^{r}(x) \cap \Delta(y)$, and suppose that $z_{I}=x_{I}^{r} y_{I}$ is nontrivial for each $I \in D$. We define an expression $Z=\prod_{I \in D} z_{I}$ (in any order). Then, if $X\left(\prod_{l \in D} x_{l}^{r}\right)$ and $\left(\prod_{l \in D} y_{I}\right) Y$ are reduced expressions for $x$ and $y$ respectively, we have that $X Z Y$ is a reduced expression for $x y$.

PROOF. Since each element $z_{I}$ is nontrivial, it is clear that both expressions $X Z$ and $Z Y$ are reduced since they are formally equivalent to the given reduced expressions for $x$ and $y$ respectively. (Two expressions $x_{1} x_{2} \cdots x_{l}$ and $y_{1} y_{2} \cdots y_{k}$ are formally equivalent if $k=l$ and $I\left(x_{i}\right)=I\left(y_{i}\right)$ for all $1 \leq i \leq l$.)

Suppose that $X Z Y=w_{1} w_{2} \cdots w_{l}$ is not reduced. Then, by Lemma 1, one may find $i<j$ such that $I\left(w_{i}\right)=I\left(w_{j}\right)$ and $I\left(w_{i}\right)$ is adjacent to $I\left(w_{k}\right)$ for all $k$ with $i<k<j$. But $w_{i}$ and $w_{j}$ are not both in $X Z$ nor both in $Z Y$, since these are reduced expressions. Thus $w_{i}$ must be from the subexpression $X$ and $w_{j}$ from $Y$. But now it follows that $w_{i}$ is a final syllable of $X Z$ and $w_{j}$ an initial syllable of $Z Y$. That is, $I\left(w_{i}\right)=I\left(w_{j}\right)=J$ for some $J \in D$. But this contradicts the fact that there is a syllable $w_{k}=z_{J}$ lying between $w_{i}$ and $w_{j}$.

\section{Quasi-lattice orders and their graph products}

Let $G$ be a group and let $P$ be a submonoid (subsemigroup containing the identity) of $G$ such that $P \cap P^{-1}=\{1\}$. Then we may define a left-invariant partial order on $G$ by $x \leq y$ whenever $x^{-1} y \in P$. Note that $x \in P$ if and only if $1 \leq x$. We observe, indeed, that every left-invariant partial order on $G$ arises in this fashion. We say that $(G, P)$ is a partially ordered group with positive cone $P$.

DEFINITION 6. A partially ordered group $(G, P)$ is quasi-lattice ordered if every finite set in $G$ with an upper bound in $G$ has a (necessarily unique) least upper bound in $G$. Equivalently, every pair $x, y$ of elements of $G$ with a common upper bound in $G$ has a least upper bound, which we denote by $x \vee y$. If $x$ and $y$ have no common upper bound in $G$, then we write $x \vee y=\infty$ for convenience.

Given the group $G$ with positive cone $P$ we may equally define a right-invariant partial order on $G$ by $x \leq_{r} y$ whenever $y x^{-1} \in P$. If $x$ and $y$ have a greatest lower bound for $\leq_{r}$, we denote it by $x \wedge_{r} y$. Clearly one has

$$
x \leq_{r} y \text { if and only if } y^{-1} \leq x^{-1}, \quad \text { and } \quad x \wedge_{r} y=\left(x^{-1} \vee y^{-1}\right)^{-1} .
$$

LEMMA 7. For a partially ordered group $(G, P)$ the following statements are equivalent.

(i) $(G, P)$ is a quasi-lattice order.

(ii) Every finite set in $G$ with a common upper bound in $P$ has a least upper bound in $P$. 
(iii) Every element $x$ of $G$ having an upper bound in $P$ has a least upper bound in $P$.

(iv) If $x \in P P^{-1}$, then there exist a pair of elements $a, b \in P$ with $x=a b^{-1}$ and such that for every $u, v \in P$ with $a b^{-1}=u v^{-1}$, one has $a \leq u$ and $b \leq v$. (The pair $a, b$ is clearly unique.)

(v) Every pair $u, v$ of elements in $P$ has a greatest lower bound $u \wedge_{r} v$ with respect to the right-invariant partial order on $G$.

(vi) If $x \in P P^{-1}$, then there exist a pair of elements $a, b \in P$ with $x=a b^{-1}$ and such that $a \wedge, b=1$.

Assuming that (i)-(vi) hold, and given $x \in P P^{-1}$, there is in fact a unique pair $a, b \in P$ satisfying statement (vi), being precisely the pair $a, b$ of statement (iv).

ProOF. We prove (i) implies (ii) implies (iii) implies (i) first. Clearly (ii) follows from (i) by observing that a (least) upper bound in $P$ for a finite set $F$ is the same thing as a (least) upper bound for $F \cup\{1\}$. Statement (iii) is obviously a special case of (ii). Finally, (iii) states that the condition of Definition 6 holds for all pairs $x, 1$, and (i) may be recovered from this by left invariance of the partial order.

(iii) implies (iv): Suppose $x \in P P^{-1}$. Then $x$ has an upper bound in $P$, and by (iii) we can take $a$ to be the least upper bound in $P$, and write $x=a b^{-1}$ for $b \in P$. If $x=u v^{-1}$ for $u, v \in P$, then $u$ is an upper bound for $x$ so we have $a \leq u$, and hence also $b \leq v\left(\right.$ since $\left.b^{-1} v=a^{-1} u \in P\right)$.

(iv) implies (v): Given $u, v \in P$, let $x=u v^{-1}$. Then the pair $a, b \in P$ of (iv) determines an element $w_{0}:=a^{-1} u=b^{-1} v$ which one checks to be the greatest right lower bound $u \wedge, v$. For if $w$ is a common right lower bound then $u=c w$ and $v=d w$, for some $c, d \in P$, and one has $c d^{-1}=u v^{-1}=a b^{-1}$. But, by (iv), both $a \leq c$ and $b \leq d$, so that $w \leq_{r} w_{0}$.

(v) implies (vi): Given $x \in P P^{-1}$, choose $u, v \in P$ such that $x=u v^{-1}$. Using (v) we may write $u=a\left(u \wedge_{r} v\right)$ and $v=b\left(u \wedge_{r} v\right)$ for some $a, b \in P$. Clearly $u v^{-1}=a b^{-1}$ and $a \wedge_{r} b=1$.

(vi) implies (iii): Let $x \in G$ have an upper bound $u$ in $P$. Then $x \in P P^{-1}$, and by (vi) we can write $x=a b^{-1}$ for $a, b \in P$ with $a \wedge_{r} b=1$, or rather $a^{-1} \vee b^{-1}=1$, using (1). Now, for any $u \in P$ that is an upper bound for $x$, we have $1 \leq u$ and $a b^{-1} \leq u$. Thus $a=a\left(a^{-1} \vee b^{-1}\right)=1 \vee a b^{-1} \leq u$. Therefore $a$ is the least upper bound of $x$ in $P$.

Finally suppose that (i)-(vi) hold and let $x=u v^{-1}$ for $u, v \in P$. Then, as in the proof of (iv) implies (v), we have $u \wedge_{r} v=a^{-1} u=b^{-1} v$ where $a, b \in P$ is the unique pair of statement (iv). Thus the pair $u, v$ satisfies the condition of statement (vi), namely $u \vee_{r} v=1$, if and only if $u=a$ and $v=b$.

REMARK 8. We make the following remarks concerning Lemma 7.

(1) Our definition of quasi-lattice order differs slightly from the one originally given 
in [18, Section 2.1], which appears here as statement (ii). Nica had also given an equivalent form of (ii) consisting of two conditions: QL1, which is statement (iii), and QL2, which is the statement of Definition 6 for all pairs $x, y \in P$. By Lemma 7 the various definitions are equivalent; in particular QL2 is not needed as it follows from QL1.

(2) In [18] and [16] least upper bounds are always assumed to be in $P$, but no such assumption is made here. This causes a slight discrepancy in notation: in this paper, the least upper bound in $P$ of $x$ and $y$ would be written $x \vee y \vee 1$.

(3) While statements (v) and (vi) may appear to be conditions only on the monoid $P$, in fact they are not, because one must have that $w \leq_{r} u \wedge_{r} v$ not only for every common right lower bound $w$ in $P$ but also for every common right lower bound $w$ in $G$.

Suppose, now, that $G=\Gamma_{I \in \Lambda} G_{I}$ is a graph product in which each group $G_{I}$, for $I \in \Lambda$, is partially ordered with positive cone $P_{l}$. We say that a reduced expression $X=x_{1} x_{2} \cdots x_{l}$ is positive if $x_{i} \in P_{I\left(x_{i}\right)}$ for all $i=1,2, \ldots, l$. Note that this property is invariant under shuffle equivalence. We say that an element $x \in G$ is positive if it has a positive reduced expression. It follows, by Theorem 2 , that every reduced expression for a positive element is positive. Let $P$ denote the submonoid of $G$ consisting of all positive elements. This is just the submonoid generated by the union of the $P_{I}$ for $I \in \Lambda$. Moreover, two positive elements are equal in $P$ if and only if their reduced expressions are shuffle equivalent. Thus $P$ may be presented as the monoid graph product $\Gamma_{l \in \Lambda} P_{l}$, that is the monoid obtained from the free product $*_{l \in \Lambda} P_{l}$ by introducing the relations $x y=y x$ for all $x \in P_{I}$ and $y \in P_{J}$ with $I$ adjacent to $J$ in $\Lambda$.

It is easily seen that $P \cap P^{-1}=\{1\}$ and hence that $(G, P)$ is a partially ordered group. We refer to $(G, P)$ as the graph product over $\Gamma$ of the partially ordered groups $\left\{\left(G_{I}, P_{I}\right)\right\}_{I \in \Lambda}$, and write $(G, P)=\Gamma_{I \in \Lambda}\left(G_{I}, P_{I}\right)$. Note that each $\left(G_{I}, P_{I}\right)$ is a partially ordered subgroup of $(G, P)$. That is, the inclusion map is order preserving.

LEMMA 9. Suppose that $(G, P)=\Gamma_{l \in \Lambda}\left(G_{l}, P_{l}\right)$ is a graph product of quasi-lattice ordered groups and denote by $\Delta^{r}(x, y)$ the intersection $\Delta^{r}(x) \cap \Delta^{r}(y)$ of the final vertex sets of elements $x$ and $y$ in $G$. For any pair $u, v \in P$, there exist $a, b \in P$ satisfying the following conditions.

(i) $a b^{-1}=u v^{-1}$, with $a \leq u, b \leq v$, and $a_{I}^{r} \wedge_{r} b_{I}^{r}=1$ for all $I \in \Delta^{r}(a, b)$. (Note that by Lemma $7(v) \wedge_{r}$ is defined in each quasi-lattice order $\left(G_{I}, P_{I}\right)$ ).

(ii) Writing reduced expressions $A \cdot\left(\prod_{\Delta^{r}(a, b)} a_{l}^{r}\right)$ and $\left(\prod_{\Delta^{r}(a, b)}\left(b_{l}^{r}\right)^{-1}\right) B$ for $a$ and $b^{-1}$ respectively, one has that

$$
A\left[\prod_{\Delta^{\prime}(a, b)}\left(a_{l}^{r}\left(b_{I}^{r}\right)^{-1}\right)\right] B
$$

is a reduced expression for $a b^{-1}$. 
PROOF. (i): We proceed by induction on $\ell(u)+\ell(v)$, the case where $u=v=1$ being trivially true. Given $u, v \in P$ we have

$$
u=\hat{u} \cdot \prod_{I \in \Delta^{r}(u, v)}\left(u_{I}^{r} \wedge_{r} v_{I}^{r}\right) \text { and } v=\hat{v} \cdot \prod_{I \in \Delta^{r}(u, v)}\left(u_{I}^{r} \wedge_{r} v_{I}^{r}\right)
$$

where $\hat{u}, \hat{v} \in P$ and $\hat{u} \hat{v}^{-1}=u v^{-1}$. Now if both $u_{I}^{r}\left(u_{I}^{r} \wedge_{r} v_{I}^{r}\right)^{-1}$ and $v_{I}^{r}\left(u_{I}^{r} \wedge_{r} v_{I}^{r}\right)^{-1}$ are nontrivial for each $I \in \Delta^{r}(a, b)$, then these are precisely the final syllables of $\hat{u}$ and $\hat{v}$ respectively (in particular, $\Delta^{r}(\hat{u}, \hat{v})=\Delta^{r}(u, v)$ ). Putting $a=\hat{u}, b=\hat{v}$ satisfies the claim in this case. Otherwise, some $u_{I}^{r} \wedge_{r} v_{I}^{r}$ equals either $u_{I}^{r}$ or $v_{I}^{r}$ for some $I \in \Delta^{r}(a, b)$. In this case $\ell(\hat{u})+\ell(\hat{v})<\ell(u)+\ell(v)$, and the result follows by induction.

(ii): This is a straightforward application of Lemma 5 , the condition $a_{l}^{r} \wedge_{r} b_{I}^{r}=1$ ensuring that the syllables $\left(a_{I}^{r}\left(b_{l}^{r}\right)^{-1}\right)$ are nontrivial.

THEOREM 10. A graph product $(G, P)=\Gamma_{I \in \Lambda}\left(G_{I}, P_{I}\right)$ of quasi-lattice ordered groups is a quasi-lattice ordered group.

ProOf. We prove that $(G, P)$ satisfies condition (iv) of Lemma 7.

Given $x \in P P^{-1}$, Lemma 9 implies that $x$ has a reduced expression $X=x_{1} x_{2} \cdots x_{m}$ in which each syllable $x_{i}$ lies in $P_{I\left(x_{i}\right)} P_{I\left(x_{i}\right)}^{-1}$. (More specifically, $X$ may take the form of (2), with the strictly positive syllables appearing first and strictly negative syllables appearing last). Since each $\left(G_{I}, P_{l}\right)$ is quasi-lattice ordered we may, in view of Lemma 7 (vi), write each $x_{i}$ uniquely as $a_{i} b_{i}^{-1}$ with $a_{i} \wedge_{r} b_{i}=1$. Define elements $\mathbf{a}, \mathbf{b} \in \boldsymbol{P}$ by $\mathbf{a}=a_{1} a_{2} \cdots a_{m}$ and $\mathbf{b}=b_{m} b_{m-1} \cdots b_{1}$. Since any shuffle of the syllables $x_{1}, x_{2}, \ldots, x_{m}$ induces a legal shuffle of the nontrivial $a_{i}$ 's (respectively the nontrivial $b_{i}$ 's) it follows by Theorem 2 that the pair of elements $\mathbf{a}, \mathbf{b}$ is uniquely determined by $x$ (independently of the choice of reduced expression).

Given any pair $u, v \in P$ with $u v^{-1}=x$, let $a, b \in P$ be any pair as in Lemma 9. The unique pair $\mathbf{a}, \mathbf{b}$ which we have just defined may be computed from any reduced expression for $x$, in particular from expression (2) of Lemma 9 (ii). It follows that $\mathbf{a}=a$ and $\mathbf{b}=b$. This shows that $x=\mathbf{a b}^{-1}$ and that $\mathbf{a} \leq u$ and $\mathbf{b} \leq v$ for every $u, v \in P$ such that $x=u v^{-1}$, as required by Lemma 7 (iv).

LEMMA 11. Suppose that $(G, P)$ is a graph product of partially ordered groups $\left(G_{l}, P_{l}\right)$ for $l \in \Lambda$. Let $x, z \in P$ be such that $1 \leq x \leq z$. Then, for each $I \in \Lambda$,

(i) $x_{I} \leq z_{l}$, and

(ii) writing $x=x_{I} x^{\prime}$, either $x_{I}=z_{l}$ or I is adjacent to every vertex of $x^{\prime}$.

Proof. We have $z=x w$, where $x, z$ and $w$ are all positive. Let $D=\Delta^{r}(x) \cap \Delta(w)$ and take reduced expressions $X\left(\prod_{J \in D} x_{J}^{r}\right)$ for $x$ and $\left(\prod_{J \in D} w_{J}\right) W$ for $w$. Note that 
$x_{J}^{r} w_{J}$ is nontrivial, for each $J \in D$, since it is a product of nontrivial positive elements. Thus, by Lemma 5 , we have the following (necessarily positive) reduced expression for $z$ :

$$
Z=X\left(\prod_{J \in D}\left(x_{J}^{r} w_{J}\right)\right) W
$$

If $z_{I}=1$ then the lemma is trivially true. Thus we suppose $z_{l} \neq 1$ and ask where the initial syllable $z_{I}$ might appear in the above expression.

Case 1. If the initial syllable $z_{I}$ falls in the subexpression $X$, then it is also an initial syllable of the reduced expression given for $x$. Thus $x_{I}=z_{l}$, satisfying (i) and (ii).

Case 2. If $I \in D$ and the initial syllable $z_{I}$ happens to be the syllable $\left(x_{I}^{r} w_{I}\right)$, then every vertex of $X$ and every $J \in D$ other than $I$ itself is adjacent to $I$. It follows that $x_{I}^{r}$ is also an initial syllable of $x$. That is $x_{I}=x_{I}^{r}$, and $x^{\prime}$ has a reduced expression

$$
X\left(\prod_{J \in D ; J \neq l} x_{J}^{r}\right)
$$

So $z_{I}=x_{I} w_{I}$ giving part (i), and $I$ is adjacent to every vertex of $x^{\prime}$ giving part (ii).

Case 3. If the initial syllable $z_{I}$ falls in the subexpression $W$, then every vertex of $x$ is adjacent to $I$ and, in particular, $x_{I}=1$. Both parts of the lemma are again satisfied.

DEFINITION 12. We consider a graph product $(G, P)=\Gamma_{J \in \Lambda}\left(G_{J}, P_{J}\right)$ of quasilattice orders, and choose $I \in \Lambda$. Given elements $x, y \in P$, write $x=x_{I} x^{\prime}$ and $y=y_{I} y^{\prime}$. We say that the elements $x, y \in P$ are $I$-adjacent if the following three conditions hold:

(a) $x_{I}$ and $y_{I}$ have a common upper bound;

(b) either $x_{I}=x_{I} \vee y_{I}$ or $I$ is adjacent to every vertex of $x^{\prime}$;

(c) either $y_{I}=x_{I} \vee y_{I}$ or $l$ is adjacent to every vertex of $y^{\prime}$.

This definition allows us to give an inductive algorithm for deciding whether two elements $x, y \in P$ have a common upper bound, and for computing $x \vee y$ when it exists. Notice first that if $x \in G_{I}$ and $y \in G_{J}$, then the least common upper bound of $x$ and $y$ in $(G, P)$ is given by

$$
x \vee y= \begin{cases}x \vee_{I} y & \text { if } I=J \\ x y & I \text { and } J \text { are adjacent; } \\ \infty & \text { if } I \text { and } J \text { are not adjacent }\end{cases}
$$


PROPOSITION 13. Suppose that $(G, P)=\Gamma_{J \in \Lambda}\left(G_{J}, P_{J}\right)$ is a graph product of quasi-lattice ordered groups. Let $x, y \in P$ and, for an arbitrary choice of $I \in \Lambda$, write $x=x_{1} x^{\prime}$ and $y=y_{1} y^{\prime}$. Then we have the following.

(i) The elements $x, y \in P$ have a common upper bound if and only if they are $I$-adjacent and $x^{\prime} \vee y^{\prime} \neq \infty$.

(ii) Suppose that the elements $x, y \in P$ do have a common upper bound. Then

$$
x \vee y=\left(x_{I} \vee y_{I}\right) \cdot\left(x^{\prime} \vee y^{\prime}\right) \text {. }
$$

Note that conditions (b) and (c) of Definition 12 apply to this expression.

PROOF. Suppose initially that the elements $x$ and $y$ are $I$-adjacent and, by condition (a) of Definition 12, write $x_{I} \vee y_{I}=x_{I} u=y_{I} v$ for some $u, v \in P_{I}$. Then, by condition (b), either $u=1$ or $I$ is adjacent to every vertex of $x^{\prime}$, in which case $x^{\prime}$ has no $I$ component. In either case one has that $u x^{\prime}=x^{\prime} u=u \vee x^{\prime}$. Similarly, by condition (c), we have $v y^{\prime}=y^{\prime} v=v \vee y^{\prime}$. Therefore, supposing in addition that $x^{\prime} \vee y^{\prime} \neq \infty$ we have:

$$
\begin{aligned}
\left(x_{I} \vee y_{I}\right)\left(x^{\prime} \vee y^{\prime}\right) & =x_{I} u x^{\prime} \vee y_{I} v y^{\prime} \\
& =x_{I}\left(u \vee x^{\prime}\right) \vee y_{I}\left(v \vee y^{\prime}\right) \\
& =\left(x_{I} \vee y_{I}\right) \vee x \vee y=x \vee y .
\end{aligned}
$$

It follows that $x$ and $y$ have a common upper bound and $x \vee y$ is given by the above equation.

On the other hand, suppose that $z=x \vee y \neq \infty$. We first observe that $x$ and $y$ are $I$-adjacent. Clearly $x_{I} \vee y_{I} \leq z$, justifying (a), and conditions (b) and (c) follow directly from Lemma 11 (ii). Finally, since $x \vee y \neq \infty$, the equalities of (3) may be read in reverse order to show that $x^{\prime} \vee y^{\prime} \neq \infty$.

Remark 14. To see that Proposition 13 leads to an effective algorithm, we note that by always choosing $I \in \Delta(x) \cup \Delta(y)$ we ensure that $\ell\left(x^{\prime}\right)+\ell\left(y^{\prime}\right)$ is always strictly less than $\ell(x)+\ell(y)$.

\section{Amenability for graph products of quasi-lattice orders}

Let $(G, P)$ be a quasi-lattice ordered group. Recall from [18] that an isometric representation $V: P \rightarrow \operatorname{Isom}(\mathscr{H})$ on a Hilbert space $\mathscr{H}$ is covariant if it is compatible with the quasi-lattice structure in the sense that

$$
V_{x} V_{x}^{*} V_{y} V_{y}^{*}=V_{x \vee y} V_{x \vee y}^{*} \quad \text { for } x, y \in P
$$


The notation is meant to include the convention $V_{\infty}=0$, so in particular covariance implies $V_{x} V_{x}^{*} V_{y} V_{y}^{*}=0$ when $x$ and $y$ have no common upper bound.

The main example of such a representation is the Toeplitz representation $T$ : $P \rightarrow \operatorname{Isom}\left(\ell^{2}(P)\right)$, defined by $T_{x} \varepsilon_{y}:=\varepsilon_{x y}$, where $\varepsilon_{x}$ denotes the typical orthonormal basis vector of $\ell^{2}(P)$. The $C^{*}$-algebra generated by the $T_{x}$ is called the Toeplitz $C^{*}$ algebra of the quasi-lattice ordered group $(G, P)$ and is denoted $\mathscr{T}(G, P)$. Nica also considered the $C^{*}$-algebra $C^{*}(G, P)$, universal for covariant isometric representations of $P$ and made the following definition.

DEFINITION 15. When the canonical homomorphism of $C^{*}(G, P)$ to $\mathscr{T}(G, P)$ is injective we say that $(G, P)$ is an amenable quasi-lattice order.

There is a semigroup $C^{*}$-dynamical system $\left(B_{P}, P, \alpha\right)$ canonically associated to $P$, in which $B_{P}$ is the $C^{*}$-subalgebra of $\ell^{\infty}(P)$ generated by the characteristic functions $1_{y}$ of the semi-infinite intervals $[y, \infty)$ for $y \in P$; the endomorphism $\alpha_{x}$ corresponding to $x \in P$ is defined by $\alpha_{x}\left(1_{y}\right)=1_{x y}$. Covariant isometric representations of $P$ are in one to one correspondence with covariant representations of the semigroup dynamical system $\left(B_{P}, P, \alpha\right)$ and this leads to the realisation of $C^{*}(G, P)$ as a semigroup crossed product, see [16, Section 2] for the details. There is a canonical conditional expectation from $B_{P} \rtimes_{\alpha} P$ onto $B_{P}$, which is faithful if and only if $(G, P)$ is amenable [18, Section 4.3]. This property, taken as the definition of amenability of $(G, P)$ in [16], is instrumental in the direct proof of amenability for free product orders, which we aim to generalise in this section.

We will need the following reformulation of Proposition 6.6 of [16].

PROPOSITION 16 (Laca, Raeburn [16]). Suppose $\phi:(G, P) \rightarrow(\mathscr{G}, \mathscr{P})$ is an order preserving homomorphism of quasi-lattice ordered groups such that, whenever $x, y \in P$ have a common upper bound in $P$,

(a) $\phi(x)=\phi(y)$ only if $x=y$, and

(b) $\phi(x) \vee \phi(y)=\phi(x \vee y)$.

If $\mathscr{G}$ is an amenable group, then $(G, P)$ is an amenable quasi-lattice order.

REMARK 17. Proposition 6.6 of [16] should have been stated like this. The reason is that the proof indicated there, modelled on that of [16, Proposition 4.2], requires that the conditional expectation for the coaction of $\mathscr{G}$ on $C^{*}(G, P)$ be faithful, which is true if $\mathscr{G}$ is amenable by [19]. We do not know whether Proposition 6.6 of [16] is correct as originally stated; however all that is required for the other results in [16] is the version stated above.

Suppose now that $(G, P)=\Gamma_{I \in \Lambda}\left(G_{l}, P_{I}\right)$ is a graph product of quasi-lattice 
ordered groups. We define the group homomorphism

$$
\phi:(G, P) \longrightarrow \bigoplus_{I \in \Lambda}\left(G_{l}, P_{l}\right)
$$

such that each factor $\left(G_{l}, P_{l}\right)$ of $(G, P)$ is mapped to the corresponding factor in the direct sum $\bigoplus_{l \in \Lambda}\left(G_{l}, P_{l}\right)$ via the identity on $G_{l}$. In what follows we shall, for simplicity, write $\phi(u)$ as $u$ whenever $u \in G_{I}$ for some $I \in \Lambda$.

We view the direct product $\bigoplus_{I \in \Lambda}\left(G_{I}, P_{I}\right)$ as a graph product (over the full graph on $\Lambda$ ). Let $x \in G$ and let $X$ be any reduced expression for $x$. Then, choosing a vertex $I \in \Lambda$, we observe that $\phi(x)_{l}$ is simply the product of all those syllables of $X$ which belong to the vertex $I$, taken in the order in which they appear. In particular, if $x=x_{I} x^{\prime}$ then $\phi(x)_{I}=x_{I} \phi\left(x^{\prime}\right)_{I}$. On the other hand, $\phi(x)_{J}=\phi\left(x^{\prime}\right)_{J}$ for all $J \neq I$.

LEMMA 18. Let $\phi$ be the map defined above. Suppose that $x, y \in P$ satisfy $x \vee y \neq \infty$ and, for an arbitrary choice of $I \in \Lambda$, write $x=x_{I} x^{\prime}$ and $y=y_{I} y^{\prime}$. Then

$$
\phi(x)_{I} \vee \phi(y)_{I}=\left(x_{I} \vee y_{I}\right) \cdot\left(\phi\left(x^{\prime}\right)_{I} \vee \phi\left(y^{\prime}\right)_{I}\right)
$$

ProOF. We refer to the final claim of Proposition 13, which states that either $y_{I} \leq x_{I}=x_{I} \vee y_{I}$ or $l$ is adjacent to every vertex of $x^{\prime}$. The latter condition implies, in particular, that $\phi(x)_{I}=x_{I}$ and $\phi\left(x^{\prime}\right)_{I}=1$. A similar statement also holds with respect to $y^{\prime}$. Thus we have the following four cases to consider:

Case 1. $\phi(x)_{I}=x_{I}$ and $\phi(y)_{I}=y_{I}$ : Since in this case $\phi\left(x^{\prime}\right)_{I}$ and $\phi\left(y^{\prime}\right)_{I}$ are both trivial, (4) is self-evident.

Case 2. $\phi(x)_{I}=x_{I}$ and $x_{I} \leq y_{I}=x_{I} \vee y_{I}$. In this case $\phi(x)_{I} \leq y_{I} \leq \phi(y)_{I}$. Also $\phi\left(x^{\prime}\right)_{I}=1$ and $x_{I} \vee y_{I}=y_{I}$. Thus (4) reduces to $\phi(y)_{I}=y_{I} \phi\left(y^{\prime}\right)_{I}$.

Case 3. $y_{I} \leq x_{I}=x_{I} \vee y_{I}$ and $\phi(y)_{I}=y_{I}$ : This case is similar to Case 2 .

Case 4. $x_{l}=y_{l}=x_{l} \vee y_{I}$ : (4) follows in this case by left invariance of the quasi-lattice order.

PROPOSITION 19. Let $(G, P)=\Gamma_{l \in \Lambda}\left(G_{l}, P_{l}\right)$ be a graph product of quasi-lattice ordered groups. Then the map $\phi:(G, P) \rightarrow \bigoplus_{I \in \Lambda}\left(G_{I}, P_{l}\right)$ (defined by the identity on each factor) is an order preserving homomorphism such that, whenever $x, y \in P$ have a common upper bound in $P$, the following hold:

(a) $\phi(x)=\phi(y)$ only if $x=y$, and

(b) $\phi(x) \vee \phi(y)=\phi(x \vee y)$.

PROOF. The induced map is clearly an order preserving homomorphism. Suppose throughout that $x, y \in P$ have a common upper bound. 
We first prove condition (a) assuming that (b) holds: Observe first of all that if $u \in P$ then $u_{I} \leq \phi(u)_{I}$ for every 1 . So $\phi(u)=1$ implies that $u=1$. Suppose that $\phi(x)=\phi(y)$. Then, by condition (b), we have

$$
\phi(x \vee y)=\phi(x) \vee \phi(y)=\phi(x)=\phi(y)
$$

Writing $x \vee y=x u=y v$ for $u, v \in P$, it follows that $\phi(u)=\phi(v)=1$. But by the preceding observation we must then have $u=v=1$ and hence $x=y$.

We now prove condition (b): Choose $I \in \Lambda$ such that either $x_{l} \neq 1$ or $y_{l} \neq 1$, and write $x=x_{I} x^{\prime}$ and $y=y_{I} y^{\prime}$. By Proposition 13 we may write $x \vee y=\left(x_{I} \vee y_{I}\right)\left(x^{\prime} \vee y^{\prime}\right)$, and hence

$$
\phi(x \vee y)=\left(x_{l} \vee y_{l}\right) \phi\left(x^{\prime} \vee y^{\prime}\right)
$$

By induction on $\ell(x)+\ell(y)$ we have that $\phi\left(x^{\prime} \vee y^{\prime}\right)=\phi\left(x^{\prime}\right) \vee \phi\left(y^{\prime}\right)$. Thus it remains to show that

$$
\phi(x) \vee \phi(y)=\left(x_{I} \vee y_{I}\right) \cdot\left(\phi\left(x^{\prime}\right) \vee \phi\left(y^{\prime}\right)\right)
$$

Note that in the direct product $\bigoplus_{J \in \Lambda}\left(G_{J}, P_{J}\right)$ every element $\xi$ may be written $\xi=\prod_{J \in \Lambda} \xi_{J}$. Combining this with Proposition 13 we have:

$$
\phi(x) \vee \phi(y)=\left(\phi(x)_{l} \vee \phi(y)_{l}\right) \prod_{J \in \Lambda ; J \neq I}\left(\phi(x)_{J} \vee \phi(y)_{J}\right) .
$$

Let $\left(6^{\prime}\right)$ denote the equation similarly obtained for the pair $x^{\prime}$ and $y^{\prime}$. We recall that $\phi(x)_{J}=\phi\left(x^{\prime}\right)_{J}$ and $\phi(y)_{J}=\phi\left(y^{\prime}\right)_{J}$ for all $J \neq I$. Thus, (5) follows from (6) and $\left(6^{\prime}\right)$ via Lemma 18.

We can now extend Theorem 6.7 of [16] to graph products of amenable groups.

THEOREM 20. Any graph product of a family of quasi-lattice orders in which the underlying groups are amenable is an amenable quasi-lattice order (that is, the Toeplitz representation is faithful).

ProOF. Let $(G, P)$ denote a graph product of the family $\left(G_{I}, P_{I}\right)_{I \in \Lambda}$ of quasilattice orders, and let $(\mathscr{G}, \mathscr{P})$ denote their direct product. By Proposition 19, the map $\phi:(G, P) \rightarrow(\mathscr{G}, \mathscr{P})$ induced by the identity on each factor satisfies the hypothesis of Proposition 16. If each $G_{I}$ is amenable as a group, then so is $\mathscr{G}$. It then follows, by Proposition 16, that $(G, P)$ is an amenable quasi-lattice order. 
As an application of Theorem 20, we obtain the following characterisation of faithfulness of representations of $C^{*}(G, P)$, and the consequent result about uniqueness of the $C^{*}$-algebra generated by a covariant isometric representation of $P$. Monoid representations $V: P \rightarrow \operatorname{Isom}(\mathscr{H})$ and $W: Q \rightarrow \operatorname{Isom}(\mathscr{H})$ are said to $*$-commute if every $V_{x}$ or $V_{x}^{*}$ for $x \in P$ commutes with every $W_{y}$ or $W_{y}^{*}$ for $y \in Q$, and to be orthogonal to one another if $V_{x}^{*} W_{y}=0$ for all $x \in P$ and $y \in Q$.

THEOREM 21. Let $(G, P)$ be the graph product of a family $\left(G_{I}, P_{l}\right)_{I \in \Lambda}$ of quasilattice ordered groups.

(i) If $\left\{V_{l}: P_{l} \rightarrow \operatorname{Isom}(\mathscr{H})\right\}_{l \in \Lambda}$ is a family of covariant isometric representations such that $V_{I} *$-commutes with $V_{J}$ when $I$ and $J$ are adjacent in $\Gamma$ and $V_{I}$ is orthogonal to $V_{J}$ when $I$ and $J$ are not adjacent in $\Gamma$, then there is a (unique) isometric covariant representation $V: P \rightarrow \operatorname{Isom}(\mathscr{H})$ such that $\left.V\right|_{P_{l}}=V_{l}$. All covariant representations of $P$ arise this way.

Suppose that each $G_{l}$, for $I \in \Lambda$, is an amenable group. Then we also have the following:

(ii) The representation of the universal algebra $C^{*}(G, P)$ associated to $V$ is faithful if and only if

$$
\prod_{x \in F}\left(1-V_{x} V_{x}^{*}\right) \neq 0 \quad \text { for every finite subset } F \subset \bigcup_{l \in \Lambda}\left(P_{I} \backslash\{1\}\right) .
$$

(iii) If $\left\{V_{l}\right\}$ and $\left\{W_{l}\right\}$ are two families of covariant isometric representations as in part (i) satisfying (7), then the canonical map $V_{I}(x) \mapsto W_{I}(x)$ extends to an isomorphism of $C^{*}\left(V_{x}: x \in P\right)$ onto $C^{*}\left(W_{x}: x \in P\right)$.

ProOF. The isometries satisfy the commuting relations which define $P$ as a graph product of monoids. Therefore the maps $s \in P_{I} \mapsto V_{I}(s)$ extend to an isometric representation $V$ of the monoid $P=\Gamma_{I \in \Lambda} P_{l}$.

We need to show that this representation is covariant, that is, we need to show that $V_{x \vee y} V_{x \vee y}^{*}=V_{x} V_{x}^{*} V_{y} V_{y}^{*}$ for all $x$ and $y$ in $P$.

We proceed by induction on $\ell(x)+\ell(y)$. Choose $I \in \Delta(x) \cup \Delta(y)$ and write $x=x_{I} x^{\prime}$ and $y=y_{I} y^{\prime}$. Since $x_{I}$ and $y_{I}$ cannot both be trivial, this ensures that $\ell\left(x^{\prime}\right)+\ell\left(y^{\prime}\right)<\ell(x)+\ell(y)$.

Suppose first that $x \vee y<\infty$. By Proposition 13 and the induction hypothesis, we have

$$
V_{x \vee y} V_{x \vee y}^{*}=V_{z} V_{x^{\prime}} V_{x^{\prime}}^{*} V_{y^{\prime}} V_{y^{\prime}}^{*} V_{z}^{*}
$$

where $z=x_{l} \vee y_{I} \in P_{I}$. Moreover, $x$ and $y$ are $l$-adjacent, and from Definition 12 (b) and (c) we have four cases to consider: 
Case 1. The vertex $I$ is adjacent to all the vertices of $x^{\prime}$ and of $y^{\prime}$. Then $V_{l} *$-commutes with $V_{x^{\prime}}$ and with $V_{y^{\prime}}$, so that (8) becomes

$$
\begin{aligned}
V_{x \vee y} V_{x \vee y}^{*} & =V_{x^{\prime}} V_{x^{\prime}}^{*} V_{z} V_{z}^{*} V_{y^{\prime}} V_{y^{\prime}}^{*} \\
& =V_{x^{\prime}} V_{x^{\prime}}^{*} V_{x_{1}} V_{x^{\prime}}^{*} V_{y_{1}} V_{y_{l}}^{*} V_{y^{\prime}} V_{y^{\prime}}^{*} \quad \text { by covariance of } V_{l} \\
& =V_{x_{1}} V_{x^{\prime}} V_{x^{\prime}}^{*} V_{x^{\prime}}^{*} V_{y_{1}} V_{y^{\prime}} V_{y^{\prime}}^{*} V_{y^{\prime}}^{*}=V_{x} V_{x}^{*} V_{y} V_{y}^{*} .
\end{aligned}
$$

Case 2. $z=x_{I}$ and $I$ is adjacent to all vertices of $y^{\prime}$. Write $z=y_{l} v$, with $v \in P_{l}$; then $V_{y^{\prime}} V_{y^{\prime}}^{*} V_{z}^{*}=V_{v}^{*} V_{y^{\prime}} V_{y^{\prime}}^{*} V_{y_{1}}^{*}=V_{z}^{*} V_{y} V_{y}^{*}$. Thus (8) becomes

$$
V_{x \vee y} V_{x \vee y}^{*}=V_{z} V_{x^{\prime}} V_{x^{\prime}}^{*} V_{z}^{*} V_{y} V_{y}^{*}=V_{x} V_{x}^{*} V_{y} V_{y}^{*}
$$

Case 3. $z=y_{I}$ and $I$ is adjacent to all vertices of $x^{\prime}$. (This is analogous to Case 2.) Case 4. $z=x_{I}=y_{I}$. Inserting $V_{z}^{*} V_{z}$ in the middle of the right-hand side of (8) we get $V_{x \vee y} V_{x \vee y}^{*}=V_{x} V_{x}^{*} V_{y} V_{y}^{*}$.

Suppose now that $x \vee y=\infty$. Then by convention $V_{x \vee y} V_{x \vee y}^{*}=0$ and it suffices to show that $V_{x}^{*} V_{y}=0$. Clearly if $x_{I} \vee y_{I}=\infty$ then, by covariance of $V_{I}$, we have $V_{x_{l}}^{*} V_{y_{l}}=0$, so $V_{x}^{*} V_{y}=0$. Thus, we may suppose that $x_{I} \vee y_{I} \neq \infty$ and hence that

$$
V_{x_{I}}^{*} V_{y_{I}}=V_{u} V_{v}^{*} \quad \text { where } x_{I} \vee y_{I}=x_{l} u=y_{I} v \text {. }
$$

Thus

$$
V_{x}^{*} V_{y}=V_{x^{\prime}}^{*} V_{u} V_{v}^{*} V_{y^{\prime}}
$$

Let $A=a_{1} a_{2} \cdots a_{k}$ be a reduced expression for $y^{\prime}$. Either condition (c) of Definition 12 holds or $v \neq 1$ and $A$ has a syllable with vertex not adjacent to $I$. Suppose the latter, and let $a_{i}$ be the first syllable of $A$ for which $I\left(a_{i}\right)$ is not adjacent to $I$. Note that $I\left(a_{i}\right) \neq I$ because $y^{\prime}$ cannot have initial vertex $I$. Write $\alpha=a_{1} a_{2} \cdots a_{i-1}$ and $\beta=a_{i+1} a_{i+2} \cdots a_{k}$. We now have

$$
V_{v}^{*} V_{y^{\prime}}=V_{v}^{*} V_{\alpha} V_{a_{i}} V_{\beta}=V_{\alpha} V_{v}^{*} V_{a_{i}} V_{\beta}=0
$$

using the fact that $V_{v}^{*} V_{a_{i}}=0$ by orthogonality.

We may thus suppose that condition (c) of Definition 12 holds, in which case $V_{v}^{*} V_{y^{\prime}}=V_{y^{\prime}} V_{v}^{*}$. By a similar argument we may suppose also that condition (b) holds and that $V_{x^{\prime}}^{*} V_{u}=V_{u} V_{x^{\prime}}^{*}$. We have already assumed that $x_{I} \vee y_{I} \neq \infty$ (part (a) of Definition 12). All these conditions together imply that $x$ and $y$ are $I$-adjacent (Definition 12) and that

$$
V_{x}^{*} V_{y}=V_{u} V_{x^{\prime}}^{*} V_{y^{\prime}} V_{v}^{*}
$$

By Proposition 13 (i) we now have $x^{\prime} \vee y^{\prime}=\infty$ (since $x \vee y=\infty$ and $x$ is $I$-adjacent to $y$ ), and applying the induction hypothesis to $x^{\prime}$ and $y^{\prime}$ completes the proof that $V$ is covariant. 
To prove (ii) observe first that from Theorem 20 and [16, Theorem 3.7], it follows that a representation is faithful if and only if

$$
\prod_{x \in F}\left(1-V_{x} V_{x}^{*}\right) \neq 0 \quad \text { for every finite subset } F \subset P .
$$

It is equivalent to consider only products of the form stated in (7) because replacing each $x \in F$ by one of its initial syllables has the effect of replacing each factor $\left(1-V_{x} V_{x}^{*}\right)$ by a smaller one. Part (iii) follows from Theorem 20 and Corollary 3.8 and Corollary 3.9 of [16].

REMARK 22. In some cases condition (7) is automatically satisfied by all covariant representations, in which case the Toeplitz $C^{*}$-algebra is simple, and purely infinite by [15, Theorem 5.4]. The best known example of this is $\theta_{\infty}$ [6]. See [16, Corollary 5.2 and Corollary 5.3] and [8, Theorem 2.4] for more examples involving free products.

\section{The $C^{*}$-algebra of a right-angled Artin semigroup of isometries}

Let $\Lambda$ be a set (usually taken to be finite, although we shall not make this restriction here). A matrix $M=\left(m_{s, t}\right)_{s, t \in \Lambda}$ is a Coxeter matrix if $m_{s, t}=m_{t, s} \in\{2,3, \ldots, \infty\}$ for $s \neq t$ and $m_{s, s}=1$. Denote by $\langle s t\rangle^{m}$ the word $s t s \cdots$, beginning with $s$ and having length $m$, in which the letters $s$ and $t$ alternate.

The Artin group $A_{M}$ associated to $M$ is the group with presentation

$$
\left.\langle\Lambda|\langle s t\rangle^{m_{s . t}}=\langle t s\rangle^{m_{s . t}} \text { for each } s, t \in \Lambda\right\rangle,
$$

in which a relation of the form $\langle s t\rangle^{\infty}=\langle t s\rangle^{\infty}$ is to be interpreted as vacuous. The Artin monoid $A_{M}^{+}$is defined via the same presentation, taken in the category of monoids (semigroups with unit), see [1]. We may view $A_{M}$ as a partially ordered group with positive cone $P_{M}$ generated by $\Lambda$. The cone $P_{M}$ is in general a quotient of $A_{M}^{+}$via the obvious map, although in many cases of interest this map is known to be injective (see [2] for the most recent results).

Adding the relations $s^{2}=1$ for $s \in \Lambda$ to the above ones yields a somewhat unusual presentation of the more familiar Coxeter group $W_{M}$ associated with $M$, which is usually presented via the relations $(s t)^{m_{s, t}}=1$.

DEFINITION 23. If the Coxeter group $W_{M}$ is finite, the associated Artin group $A_{M}$ is said to be of finite type, [1].

A Coxeter group and its associated Artin group $A_{M}$ are said to be right-angled if every nondiagonal entry of the Coxeter matrix $M$ is either 2 or $\infty$; see for example [3]. (By abuse we also refer to the matrix as right-angled.) 
The terminology is motivated by noting that the right-angled Coxeter groups are those linear reflection groups whose reflecting hyperplanes are mutually orthogonal or parallel.

Every right-angled Coxeter matrix $M$ over $\Lambda$ determines a graph $\Gamma$ with vertex set $\Lambda$ having an edge joining $s$ and $t$ when $m_{s, t}=2$. The only relations in the presentation of $A_{M}$ say that two generators commute if they are joined by an edge, hence $A_{M}$ is precisely the graph product $\Gamma_{l \in \Lambda} \mathbb{Z}$ of copies of $\mathbb{Z}$. In this connection, right-angled Artin groups are also referred to as graph groups, see [14].

By virtue of Theorem 2 the right-angled Artin monoid $A_{M}^{+}$may be identified with the positive cone of the corresponding Artin group $A_{M}$, and $\left(A_{M}, A_{M}^{+}\right)$is a quasi-lattice order by Theorem 10. Applying Theorem 20 we see that this quasi-lattice order is amenable, and hence the Toeplitz representation of $C^{*}\left(A_{M}, A_{M}^{+}\right)$is faithful. As with Coburn's and Cuntz's theorems, it is more appealing to formulate the result in terms of the generators themselves; indeed, notice that assertion (iii) below does not contain any explicit reference to quasi-lattice orders or Artin groups.

THEOREM 24. Let $\Gamma$ be a graph with set of vertices $\Lambda$ and suppose $\left\{V_{s}: s \in \Lambda\right\}$ is a collection of isometries on Hilbert space such that for every pair of distinct vertices $s$ and $t$ one has

$$
\begin{aligned}
& V_{s} V_{t}=V_{t} V_{s} \quad \text { and } \quad V_{s}^{*} V_{t}=V_{t} V_{s}^{*} \quad \text { if } s \text { and } t \text { are adjacent in } \Gamma \text {, and } \\
& V_{s}^{*} V_{t}=0 \text { if } s \text { and } t \text { are not adjacent in } \Gamma \text {. }
\end{aligned}
$$

Let $A_{M}=\Gamma_{s \in \Lambda} \mathbb{Z}\langle s\rangle$ be the right-angled Artin group associated to $\Gamma$. Then

(i) the maps $s \rightarrow V_{s}$, for $s \in \Lambda$, extend to a covariant isometric representation $V$ of the right-angled Artin semigroup $A_{M}^{+}$,

(ii) the corresponding representation of $C^{*}\left(A_{M}, A_{M}^{+}\right)$is faithful if and only if

$$
\prod_{s \in F}\left(I-V_{s} V_{s}^{*}\right) \neq 0 \quad \text { for every finite } F \subset \Lambda, \quad \text { and }
$$

(iii) if $\left\{W_{s}: s \in \Lambda\right\}$ is another collection of isometries satisfying the same relations and condition (9), then the map $V_{s} \mapsto W_{s}$ extends to a $C^{*}$-algebra isomorphism of $C^{*}\left(V_{s}: s \in \Lambda\right)$ to $C^{*}\left(W_{s}: s \in \Lambda\right)$.

PROOF. Since the generators $V_{s}$ satisfy the stated relations, the collection of isometric representations $\left\{n \in \mathbb{N} \mapsto V_{s}^{n}\right\}_{s \in \Lambda}$ satisfies the hypothesis of Theorem 21 (i) and so extends to an isometric representation of the semigroup $A_{M}^{+}$, giving (i). The amenability hypothesis is satisfied because in this case each factor in the graph product is isomorphic to $\mathbb{Z}$, so (ii) and (iii) follow directly from Theorem 21 . Notice that the 
necessary and sufficient condition for faithfulness in (ii) is equivalent to the one obtained in Theorem 21 because every projection of the form (7) majorates a projection of the form

$$
\prod_{s \in F}\left(I-V_{s} V_{s}^{*}\right) \quad \text { for some finite } F \subset \Lambda,
$$

to see this it suffices to replace each syllable $x=s^{n} \in \mathbb{N}\langle s\rangle$ by the corresponding generator $s$.

REMARK 25. If the set $\Lambda$ of generators is finite, the projection $\prod_{s \in \Lambda}\left(I-T_{s} T_{s}^{*}\right)$ belongs to the Toeplitz algebra $\mathscr{T}\left(A_{M}, A_{M}^{+}\right)$. Since every nontrivial element in $A_{M}^{+}$is bounded below by a generator, it follows from [18, Proposition 6.3] that the compact operators on $\ell^{2}(P)$ are contained in $\mathscr{T}\left(A_{M}, A_{M}^{+}\right)$as the ideal generated by this projection. It is easy to see that (9) holds if and only if it holds for $F=\Lambda$, so this ideal is minimal.

\section{Non-amenability of lattice ordered groups}

Next we concentrate on partially ordered groups in which least common upper bounds always exist.

DEFINITION 26. A partially ordered group $(G, P)$ is lattice ordered if every pair of elements has a least common upper bound. By left invariance, an equivalent condition is that every element $x \in G$ have a least upper bound in $P$.

Lattice orders are special cases of quasi-lattice orders; in fact we have the following characterisation, see for example [18].

LEMMA 27. The following are equivalent for a partially ordered group $(G, P)$ :

(i) $(G, P)$ is lattice ordered.

(ii) $(G, P)$ is quasi-lattice ordered and $G=P P^{-1}$.

(iii) $(G, P)$ is quasi-lattice ordered, $P$ generates $G$ (in which case we say $(G, P)$ is connected), and $a P \cap b P \neq \emptyset$ for all $a, b \in P$.

ProOF. (i) implies (ii): Suppose $(G, P)$ is lattice ordered and let $x \in G$; then $x \leq x \vee 1$ so that both $a:=x \vee 1$ and $b:=x^{-1} a$ are in $P$. Clearly $x=a b^{-1}$.

(ii) implies (iii): If $G=P P^{-1}$ then obviously $P$ generates $G$. Given a pair $a, b \in P$, write $a^{-1} b$ as $x y^{-1}$ with $x, y \in P$. Then $a x=b y \in a P \cap b P$.

(iii) implies (i): Suppose (iii) holds. Take any $x \in G$ and (using the connectedness) write $x=a_{1} a_{2} \cdots a_{k}$ with each $a_{i} \in P$ or $P^{-1}$. Since any element $a^{-1} b$ for $a, b \in P$ 
may be rewritten $c d^{-1}$ for $c, d \in P$ (by finding $a c=b d \in a P \cap b P$ ), any such expression for $x$ may ultimately be simplified to the form $x=u v^{-1}$ with $u, v \in P$. Thus $x$ has an upper bound $u \in P$ and, since $(G, P)$ is a quasi-lattice order, it must have a least upper bound in $P$. So (i) holds.

For a quasi-lattice order $(G, P)$, we know that if $G$ is amenable then $(G, P)$ is amenable, by [18, Section 4.5], see also [16, Lemma 6.5]. It turns out that for lattice ordered groups the converse is also true; the proof follows the argument outlined in Remark 2 of [18, Section 5.1].

PROPOSITION 28. If $(G, P)$ is lattice ordered and amenable (in the sense of Nica, Definition 15$)$ then $G$ is an amenable group.

Proof. Suppose $(G, P)$ is an amenable lattice order, and denote the left regular representation of $P$ on $\ell^{2}(P)$ by $W$. As observed by Nica, the map $x \in P \mapsto 1 \in \mathrm{C}$ is a (one-dimensional) covariant isometric representation, so the map $W_{x} \mapsto 1$ extends to give a one-dimensional representation of $\mathscr{T}(G, P)$, and an easy argument shows that

$$
\left\|\sum_{s \in P} \lambda_{s} W_{s}\right\|=\sum_{s \in P} \lambda_{s}
$$

for every finitely (or countably) supported nonnegative function $\lambda$ in $\ell^{1}(P)$.

Notice that the sum $\sum_{s} \lambda_{s} W_{s}$ is the operator of left-convolution by $\lambda \in \ell^{1}(P)$ on $\ell^{2}(P)$, and that (10) implies that $\left\|\sum_{s \in P} \lambda_{s} W_{s}\right\|=1$ for every probability density $\lambda$. Since the support of $\lambda$ can be chosen to contain an arbitrary finite subset of $P$ including the identity, we have that condition (e) of $[9$, Theorem 1] holds, with $p=2$, $U=1 \in P$, and $\phi$ a probability density whose support contains $\xi \cup\{1\}$. By [9, Theorem 1], the semigroup $P$ has a left-invariant mean. (Day's Theorem is about right-amenability and right-convolutions, but there is no difficulty in transforming it into a theorem for left-amenability and left-convolutions.)

Finally, the group $G=P P^{-1}$ is amenable, by Corollary 3.6 of [21].

By Proposition 5.5 and Theorem 5.6 of [1], if $A_{M}$ is of finite type then the Artin semigroup $A_{M}^{+}$embeds as a subsemigroup of $A_{M}$ and the pair $\left(A_{M}, A_{M}^{+}\right)$is a lattice ordered group (see also [10]). We wish to apply Proposition 28 to characterize amenability of these lattice orders; the first observation is that most Artin groups are not amenable.

Proposition 29. Let $M$ be a Coxeter matrix over a finite set $\Lambda$. The Artin group $A_{M}$ is amenable (as a group) if and only if it is the free abelian group on $\Lambda$, that is, $m_{s, t}=2$ for all $s, t \in \Lambda, s \neq t$. 
PROOF. In [5] it is shown that the set of elements $\mathscr{Q}=\left\{Q_{s}=s^{2}: s \in \Lambda\right\}$ generates a subgroup $H_{M}$ of $A_{M}$ with presentation

$$
\left.\langle\mathscr{Q}| Q_{s} Q_{t}=Q_{t} Q_{s} \text { if } m_{s, t}=2\right\rangle
$$

That is, $H_{M}$ is a right-angled Artin group. In particular, $H_{M}$ contains at least one free subgroup of rank 2 , and hence is not amenable, unless of course $m_{s, t}=2$ for all $s \neq t \in \Lambda$. In the latter case $A_{M}$ is free abelian and therefore amenable.

As a consequence of Proposition 29, the analog of Theorem 21 (ii) fails for nonabelian Artin groups of finite type, instead we have the following non-uniqueness result.

THEOREM 30. Let $A_{M}$ be a nonabelian Artin group of finite type. Suppose $\left\{V_{s}\right.$ : $s \in \Lambda\}$ is a collection of isometries satisfying the Artin relations relative to $M$ :

$$
\left\langle V_{s} V_{t}\right\rangle^{m_{s, t}}=\left\langle V_{t} V_{s}\right\rangle^{m_{s, t}} \quad s, t \in \Lambda
$$

Then the map $s \mapsto V_{s}$ extends to an isometric representation, denoted also by $V$, of the Artin semigroup $A_{M}^{+}$. The representation $V$ is covariant provided that

$$
V_{s} V_{s}^{*} V_{t} V_{t}^{*}=V_{s \vee t} V_{s \vee t}^{*} \quad s, t \in \Lambda \text {. }
$$

The Toeplitz representation $T$ on $\ell^{2}\left(A_{M}^{+}\right)$satisfies (12) and (13) and, moreover, the projection $\prod_{\Lambda}\left(1-T_{s} T_{s}^{*}\right)$ does not vanish. However, the Toeplitz representation of $C^{*}\left(A_{M}, A_{M}^{+}\right)$is not faithful; in particular, the $C^{*}$-algebra generated by a collection $\left\{V_{s}: s \in \Lambda\right\}$ as above is not canonically unique, even if we assume $\prod_{\Lambda}\left(1-V_{s} V_{s}^{*}\right) \neq 0$.

PROOF. The map can be extended to an isometric representation because the given isometries satisfy the relations (12), which constitute a presentation of $A_{M}^{+}$.

Suppose that (13) holds. We need to show that the covariance condition holds for every pair $x, y \in A_{M}^{+}$. We use the length homomorphism $l: A_{M}^{+} \rightarrow \mathbb{N}$ such that $l(s)=1$ for each generator $s \in \Lambda$. Choose $x, y$ such that the covariance condition is not satisfied and such that $l(x \vee y)$ is minimised. Amongst the possible pairs, choose one so that $l(x)$ is the smallest possible.

Case 1. Suppose $l(x)=1$, so that $x$ is actually a generator $s$. Write $y=t z$ for $t$ a generator, so that $V_{y} V_{y}^{*}=V_{t}\left(V_{t}^{*} V_{t}\right) V_{z} V_{y}^{*}=V_{t} V_{t}^{*} V_{y} V_{y}^{*}$. Then, by (13),

$$
V_{s} V_{s}^{*} V_{y} V_{y}^{*}=V_{s} V_{s}^{*} V_{t} V_{t}^{*} V_{y} V_{y}^{*}=V_{s \vee t} V_{s \vee t}^{*} V_{y} V_{y}^{*}
$$

Now $s \vee t$ and $y$ have a common left factor $t$; writing $s \vee t=t u$ and $y=t z$ we have

$$
V_{s \vee t} V_{s \vee t}^{*} V_{y} V_{y}^{*}=V_{t} V_{u} V_{u}^{*} V_{z} V_{z}^{*} V_{t}^{*}
$$


Since $s \vee y=s \vee t \vee y=t(u \vee z)$, we have that $l(u \vee z)<l(s \vee y)$ and covariance follows by applying the induction hypothesis to $V_{u} V_{u}^{*} V_{z} V_{z}^{*}$ in this expression.

Case 2. Suppose now $l(x)>1$, and write $x=s u$ for $s$ a generator. Put $s \vee y=s z$, so that $x \vee y=x \vee s \vee y=s(u \vee z)$. Since $l(s \vee y) \leq l(x \vee y)$ and $l(s)<l(x)$, the induction hypothesis implies that $V_{s} V_{s}^{*} V_{y} V_{y}^{*}=V_{s} V_{z} V_{z}^{*} V_{s}^{*}$. Applying $V_{s}^{*}$ on the left and using the fact that $V_{s}^{*} V_{s}=1$ this equation becomes $V_{s}^{*} V_{y} V_{y}^{*}=V_{z} V_{z}^{*} V_{s}^{*}$. We then have: $V_{x} V_{x}^{*} V_{y} V_{y}^{*}=V_{s} V_{u} V_{u}^{*} V_{s}^{*} V_{y} V_{y}^{*}=V_{s} V_{u} V_{u}^{*} V_{z} V_{z}^{*} V_{s}^{*}$. Since $x \vee y=s(u \vee z)$ we have $l(u \vee z)<l(x \vee y)$, and covariance follows in this case, as before, by applying the induction hypothesis to $V_{u} V_{u}^{*} V_{z} V_{z}^{*}$.

It is clear that the Toeplitz representation satisfies the stated relations, and the projection $\prod_{\Lambda}\left(1-T_{s} T_{s}^{*}\right)$ does not vanish at $\delta_{1} \in \ell^{2}\left(A_{M}^{+}\right)$. The quasi-lattice ordered group $\left(A_{M}, A_{M}^{+}\right)$is not amenable by Proposition 28 and Proposition 29 and the last assertion follows, see [16, Corollary 3.9$]$.

REMARK 31. Since Theorem 30 shows that the Toeplitz algebra $\mathscr{T}\left(A_{M}, A_{M}^{+}\right)$of a nonamenable finite type Artin group $A_{M}$ is not universal for covariant isometric representations, it is generally hard to decide whether a given collection of isometries satisfying the Artin relations and the covariance condition actually generates a representation of $\mathscr{T}\left(A_{M}, A_{M}^{+}\right)$. In any case, it follows from Theorem 6.7 and Corollary 6.8 of [12] that a given a representation of $\mathscr{T}\left(A_{M}, A_{M}^{+}\right)$is faithful if and only if the generating family of isometries is proper, in the sense that $\prod_{\Lambda}\left(1-V_{s} V_{s}^{*}\right) \neq 0$.

Our results about Toeplitz algebras cover the Artin groups $\left(A_{M}, A_{M}^{+}\right)$that are presently known to be quasi-lattice ordered, namely the finite type Artin groups from $[1,10]$ and the right-angled Artin groups dealt with by Theorem 10 . It would be interesting to formulate and decide questions of amenability and uniqueness in the remaining cases. It is known that, for $\Lambda$ finite, the monoid $A_{M}^{+}$always has a quasilattice structure (see $[1,10]$ ), but even when it is known that $A_{M}^{+}$embeds canonically in $A_{M}$ this is not enough to show that $\left(A_{M}, A_{M}^{+}\right)$is quasi-lattice ordered, which is essential for our techniques.

Note added in proof. At the time this paper was written the best known results on the 'injectivity of the Artin monoid' were contained in [2]. Since then, the proof that $A_{M}^{+}$embeds canonically in $A_{M}$ for any Coxeter matrix $M$ has been announced by Luis Paris in a preprint entitled 'Artin monoids inject in their groups'.

\section{References}

[1] E. Brieskom and K. Saito, 'Artin-Gruppen und Coxeter-Gruppen', Invent. Math. 17 (1972), 245271. 
[2] R. Charney, 'Injectivity of the positive monoid for some infinite type Artin groups', in: Geometric group theory down under. Proceedings of a special year in geometric group theory, Canberra, Australia, 1996, (eds. J. Cossey, et al.), (W. de Gruyter, Berlin, 1999) pp. 103-118.

[3] I. M. Chiswell, 'Right-angled Coxeter groups', in: Low dimensional topology and Kleinian groups (ed. D. B. A. Epstein), London Math. Soc. Lecture Note Ser. 112, (Cambridge University Press, Cambridge, 1986) pp. 297-304.

[4] L. A. Coburn, 'The $C^{*}$-algebra generated by an isometry I', Bull. Amer. Math. Soc. 73 (1967), $722-726$.

[5] J. Crisp and L. Paris, 'The solution to a conjecture of Tits on the subgroup generated by the squares of the generators of an Artin group', Invent. Math. 145 (2001), 19-36.

[6] J. Cuntz, 'Simple $C^{*}$-algebras generated by isometries', Comm. Math. Phys. 57 (1977), 173-185.

[7] — 'K-theory for certain $C^{*}$-algebras', Ann. of Math. (2) 113 (1981), 181-197.

[8] K. R. Davidson and G. Popescu, 'Noncommutative disc algebras for semigroups', Canad. J. Math. 50 (1998), 290-311.

[9] M. M. Day, 'Amenable semigroups', Illinois J. Math. 8 (1964), 100-111.

[10] P. Deligne, 'Les immeubles des groupes de tresses généralisés', Invent. Math. 17 (1972), 273-302.

[11] R. G. Douglas, 'On the $C^{*}$-algebra of a one-parameter semigroup of isometries', Acta Math. 128 (1972), 143-152.

[12] R. Exel, M. Laca and J. C. Quigg, 'Partial dynamical systems and $C^{*}$-algebras generated by partial isometries', J. Operator Theory, to appear.

[13] E. R. Green, Graph products of groups (PhD Thesis, The University of Leeds, 1990).

[14] S. Hermiller and J. Meier, 'Algorithms and geometry for graph products of groups', J. Algebra 171 (1995), 230-257.

[15] M. Laca, 'Purely infinite simple Toeplitz algebras', J. Operator Theory 41 (1999), 421-435.

[16] M. Laca and I. Raeburn, 'Semigroup crossed products and the Toeplitz algebras of nonabelian groups', J. Funct. Anal. 139 (1996), 415-440.

[17] G. J. Murphy, 'Ordered groups and Toeplitz algebras', J. Operator Theory 18 (1987), 303-326.

[18] A. Nica, ' $C$-algebras generated by isometries and Wiener-Hopf operators', J. Operator Theory 27 (1992), 17-52.

[19] J. C. Quigg, 'Full and reduced $C^{*}$-coactions', Math. Proc. Cambridge Philos. Soc. 116 (1994), 435-450.

[20] H. Salas, 'Semigroups of isometries with commuting range projections', J. Operator Theory 14 (1985), 311-346.

[21] C. Wilde and K. Witz, 'Invariant means and the Stone-Cech compactification', Pacific J. Math. 21 (1967), 577-586.

Laboratoire de Topologie

Université de Bourgogne

UMR 5584 du CNRS

B.P. 47870

21078 Dijon Cedex

France

e-mail: crisp@topolog.u-bourgogne.fr
Department of Mathematics The University of Newcastle

NSW 2308

Australia

Current address: Mathematisches Institut Westfälische Wilhelms-Universität Einsteinstr. 62 48149 Münster

Germany

e-mail: laca@math.uni-muenster.de 
